\title{
Case Report \\ Detection of an Ambler class D OXA-48-type $\beta$-lactamase in a Klebsiella pneumoniae strain in The Netherlands
}

\begin{abstract}
Correspondence
Jayant S. Kalpoe

jayant.kalpoe@slz.nl
\end{abstract}

Received 20 November 2010

Accepted 19 January 2011

\author{
Jayant S. Kalpoe, ${ }^{1} \dagger$ Nashwan Al Naiemi, ${ }^{2}$ Laurent Poirel $^{3}$ \\ and Patrice Nordmann ${ }^{3}$ \\ ${ }^{1}$ Department of Medical Microbiology and Infection Prevention, Saint Lucas Andreas Hospital, \\ Amsterdam, The Netherlands \\ ${ }^{2}$ Microbiology Laboratory Twente Achterhoek, Enschede, The Netherlands \\ ${ }^{3}$ Service de Bactériologie-Virologie-Hygiène, Hôpital de Bicêtre, Le Kremlin-Bicêtre, France
}

Traditionally, bacteria in The Netherlands have low levels of resistance to antibiotics. This report describes what is believed to be the first carbapenem-resistant Klebsiella pneumoniae producing an OXA-48 type $\beta$-lactamase in The Netherlands. The isolate co-produced a CTX-M-15 type $\beta$-lactamase and was recovered from a patient who was transferred from a hospital in India to an intensive care unit in The Netherlands. His recovery in The Netherlands was complicated by pneumonia due to the carbapenem-resistant $K$. pneumoniae to which he eventually succumbed. Pre-emptive screening for carbapenem-resistant Enterobacteriaceae in selected patients could be imperative to maintain the low prevalence of these highly resistant bacteria in Dutch hospitals.

\section{Introduction}

Carbapenems are the drugs of last resort, especially for infection with extended-spectrum $\beta$-lactamase-producing Enterobacteriaceae, which are often resistant to fluoroquinolones, aminoglycosides, trimethoprim-sulfamethoxazole (co-trimoxazole) and other antibiotics. The emergence and ongoing global dissemination of carbapenem-resistant Enterobacteriaceae impose a significant threat to healthcare facilities. Carbapenem resistance in Enterobacteriaceae may be caused by the combined mechanisms of an outermembrane permeability defect and extended-spectrum $\beta$-lactamases or hyperproduction of AmpC and upregulation of efflux systems (Bradford et al., 1997), but is mostly due to the production of carbapenem-hydrolysing $\beta$-lactamases (carbapenemases) (Poirel et al., 2007). Carbapenemases can be metallo- $\beta$-lactamases (Ambler class B), Klebsiella pneumoniae carbapenemases or KPC (Ambler class A), or more rarely carbapenem-hydrolysing oxacillinases of the OXA-48-type (Ambler class D) (Poirel et al., 2007). The carbapenem-hydrolysing class D $\beta$-lactamase OXA-48 was first identified in a K. pneumoniae isolate from Turkey (Poirel et al., 2004). Since then, several other OXA-48-producing isolates of various enterobacterial species (Citrobacter freundii and Escherichia coli) have been reported, mainly from Turkey but also from other countries, including

tPresent address: Department of Medical Microbiology, Slotervaart Hospital/Dutch Cancer Institute, 1006 BK Amsterdam, The Netherlands.

Abbreviation: ICU, intensive care unit.
Belgium (Cuzon et al., 2008a), the UK (Livermore, 2009) and India (Bell et al., 2009). Here we describe a case of what is believed to be the first carbapenem resistant OXA-48-type producing $K$. pneumoniae isolate from The Netherlands.

\section{Case report}

In March 2010, a 63-year-old Dutch man was transferred from a hospital in New Delhi, India, to an intensive care unit (ICU) in Amsterdam, The Netherlands. During his vacation in New Delhi, he was admitted to a local ICU after surgical intervention to resolve a tension pneumothorax. Upon arrival in Amsterdam, surveillance cultures were performed to screen for multi-resistant bacteria and contact precautions were installed. The first surveillance culture of sputum showed a K. pneumoniae isolate resistant to broad-spectrum cephalosporins, meropenem, ertapenem and imipenem as determined by the Vitek-2 system (bioMérieux), the Phoenix microbiology system (Becton Dickinson) and Etest (AB Biodisk) according to European Committee on Antimicrobial Susceptibility Testing criteria. In addition the isolate was also resistant to fluoroquinolones, co-trimoxazole, tobramycin and gentamicin, and remained susceptible only to tigecycline and colistin (Table 1). Since the patient showed no signs of infection, antibiotic treatment was not initiated at this time. Surveillance cultures were continued biweekly and the multi-resistant K. pneumoniae was repeatedly recovered from respiratory surveillance cultures. 
Table 1. MICs of antibiotics for K. pneumoniae (AMS-1)

\begin{tabular}{|lcc|}
\hline Antibiotic & Susceptibility & $\mathbf{M I C}\left(\boldsymbol{\mu g} \mathbf{~ m l}^{-\mathbf{1}}\right)$ \\
\hline Amoxicillin & $\mathrm{R}$ & $>32$ \\
Amoxicillin + clavulanic acid & $\mathrm{R}$ & $>32$ \\
Cefuroxime & $\mathrm{R}$ & $>64$ \\
Ceftriaxone & $\mathrm{R}$ & $>64$ \\
Ceftazidime & $\mathrm{R}$ & $>64$ \\
Gentamicin & $\mathrm{R}$ & $>16$ \\
Tobramycin & $\mathrm{R}$ & $>16$ \\
Meropenem & $\mathrm{R}$ & $>16$ \\
Imipenem & $\mathrm{R}$ & 8 \\
Ertapenem & $\mathrm{R}$ & $>16$ \\
Ciprofloxacin & $\mathrm{R}$ & $>4$ \\
Co-trimoxazole & $\mathrm{R}$ & $>320$ \\
Colistin & $\mathrm{S}$ & 1 \\
Tigecycline & $\mathrm{S}$ & 1 \\
\hline
\end{tabular}

R, Resistant; S, susceptible.

PCR and sequence analysis with primers for detection of Ambler class D and class B, and class A $\beta$-lactamaseencoding genes (Cuzon et al., 2008b), identified an OXA48-type $\beta$-lactamase together with the $b l a_{\mathrm{CTX}-\mathrm{M}-15}$ genes. Sequencing identified a variant of the class D carbapenemase OXA-48 (differing by 4 amino acid substitutions) that corresponds to the carbapenemase OXA-181. The latter enzyme was recently identified from Indian isolates (Castanheira et al., 2011; Poirel et al., 2011). Four weeks after his transfer from New Delhi, the patient developed pneumonia, with surveillance cultures as well as diagnostic sputum cultures positive for the OXA-48-producing Klebsiella, upon which simultaneous treatment with intravenous tigecycline (starting dose $100 \mathrm{mg}$, followed by $50 \mathrm{mg}$ twice daily) and intravenous colistin (160 mg three times daily) was started. The infection was soon resolved and the patient was moved to the pulmonary ward where he repeatedly developed pulmonary distress, presumably sustained by the multi-resistant Klebsiella to which he eventually succumbed, 40 days after his admission to the ICU. No other patients with carbapenem-resistant Enterobacteriaceae were identified in the hospital at the time of his admittance and during the case. Carbapenemases of the OXA-48 type have been described in patients in India (Bell et al., 2009). This is believed to be the first evidence of an OXA-48-type $\beta$ lactamase-producing Enterobacteriaceae in The Netherlands, in a patient transferred from an Indian ICU to Amsterdam.

\section{Conclusion}

Traditionally, The Netherlands has low levels of bacterial resistance to antibiotics and no major outbreaks with carbapenemase-producing Enterobacteriaceae have been reported yet. Therefore, it is of great relevance to maximize efforts to prevent or slow down the dissemination of carbapenemase-producing bacteria in Dutch hospitals, when the presumed prevalence of such bacteria is still among the lowest in Europe. Pre-emptive screening for carbapenemresistant Enterobacteriaceae in patients transferred from high incidence areas or from any foreign hospital could be imperative to maintain this low prevalence.

\section{References}

Bell, J. M., Mathai, D., Jones, R. N. \& Turnidge, J. D. (2009). Emergence of OXA-48 carbapenemases among Klebsiella spp. from India. In Abstracts of the 49th Interscience Conference on Antimicrobial Agents and Chemotherapy (ICAAC), abstract C2-650. Washington, DC: American Society for Microbiology.

Bradford, P. A., Urban, C., Mariano, N., Projan, S. J., Rahal, J. J. \& Bush, K. (1997). Imipenem resistance in Klebsiella pneumoniae is associated with the combination of ACT-1, a plasmid-mediated AmpC $\beta$-lactamase, and the loss of an outer membrane protein. Antimicrob Agents Chemother 41, 563-569.

Castanheira, M., Deshpande, L. M., Mathai, D., Bell, J. M., Jones, R. N. \& Mendes, R. E. (2011). Early dissemination of NDM-1- and OXA181-producing Enterobacteriaceae in Indian hospitals: report from the SENTRY Antimicrobial Surveillance Program (2006-2007). Antimicrob Agents Chemother (in press).

Cuzon, G., Naas, T., Bogaerts, P., Glupczynski, Y., Huang, T. D. \& Nordmann, P. (2008a). Plasmid-encoded carbapenem-hydrolyzing $\beta$ lactamase OXA-48 in an imipenem-susceptible Klebsiella pneumoniae strain from Belgium. Antimicrob Agents Chemother 52, 3463-3464.

Cuzon, G., Naas, T., Demachy, M. C. \& Nordmann, P. (2008b). Plasmid-mediated carbapenem-hydrolyzing $\beta$-lactamase KPC-2 in Klebsiella pneumoniae isolate from Greece. Antimicrob Agents Chemother 52, 796-797.

Livermore, D. M. (2009). Has the era of untreatable infections arrived? J Antimicrob Chemother 64 (Suppl. 1), i29-i36.

Poirel, L., Héritier, C., Tolün, V. \& Nordmann, P. (2004). Emergence of oxacillinase-mediated resistance to imipenem in Klebsiella pneumoniae. Antimicrob Agents Chemother 48, 15-22.

Poirel, L., Pitout, J. D. \& Nordmann, P. (2007). Carbapenemases: molecular diversity and clinical consequences. Future Microbiol 2, 501-512.

Poirel, L., Ros, A., Carricajo, A., Berthelot, P., Pozzetto, B., Bernabeu, S. \& Nordmann, P. (2011). Extremely drug-resistant Citrobacter freundii isolate producing NDM-1 and other carbapenemases identified in a patient returning from India. Antimicrob Agents Chemother 55, 447448. 